\title{
S100A9 promotes the proliferation and invasion of HepG2 hepatocellular carcinoma cells via the activation of the MAPK signaling pathway
}

\author{
RUI WU ${ }^{1 *}$, LIANG DUAN $^{1 *}$, LIWEI YE ${ }^{1}$, HAIYANG WANG ${ }^{1}$, XIA YANG $^{1}$, YUNYUAN ZHANG $^{1}$, \\ XIAN CHEN $^{1}$, YAN ZHANG ${ }^{1}$, YAGUANG WENG ${ }^{1}$, JINGYONG LUO ${ }^{1}$, MIN TANG ${ }^{1}$, \\ QIONG SHI ${ }^{1}$, TONGCHUAN HE ${ }^{2}$ and LAN ZHOU ${ }^{1}$ \\ ${ }^{1}$ Department of Laboratory Medicine, Chongqing Medical University, Chongqing 400016, P.R. China; \\ ${ }^{2}$ Molecular Oncology Laboratory, Department of Surgery, The University of Chicago \\ Medical Center, Chicago, IL 60637, USA
}

Received November 7, 2012; Accepted January 4, 2013

DOI: $10.3892 /$ ijo.2013.1796

\begin{abstract}
The S100A9 protein, a member of the S100 protein family, is often upregulated in various types of cancer, including hepatocellular carcinoma (HCC). S100A9 acts as a danger signal when secreted to the extracellular space and is thought to play an important role during tumorigenesis. Despite this fact, little is known about the effects of S100A9 in the tumor microenvironment on HCC. Therefore, in this study, we investigated the effects of exogenous S100A9 on the proliferation and invasion of HepG2 HCC cells, as well as the molecular mechanisms underlying these effects. Our results demonstrated that exogenous S100A9 promoted the proliferation, clone formation and invasion of HepG2 cells in vitro, as shown by 3-(4,5-dimethylthiazol-2-yl)-2,5-diphenyltrazolium bromide (MTT), clone formation and transwell invasion assays, respectively, and also promoted tumor growth in vivo by tumorigenicity assays in nude mice. Furthermore, S100A9 increased the phosphorylation of p38 and ERK1/2 mitogen-activated protein kinases (MAPKs) in HepG2 cells. When the phosphorylation of p38 was inhibited by SB203580 (a p38 inhibitor), the S100A9induced cell invasion was reversed; when the phosphorylation of ERK1/2 was inhibited by PD98059 (an ERK1/2 inhibitor), the S100A9-induced cell proliferation was reversed. These data suggest that the S100A9-induced proliferation and invasion of HepG2 cells are partly mediated by the activation of the MAPK signaling pathway.
\end{abstract}

Correspondence to: Dr Lan Zhou, Department of Laboratory Medicine, Chongqing Medical University, 1 Yixueyuan Road, Yuzhong District, Chongqing 400016, P.R. China

E-mail: zhoulan0111@foxmail.com

${ }^{*}$ Contributed equally

Key words: hepatocellular carcinoma, S100A9, mitogen-activated protein kinase, proliferation, invasion

\section{Introduction}

Hepatocellular carcinoma (HCC) is one of the most common types of tumor worldwide, with a high mortality rate (1). Risk factors for HCC development include infection with hepatitis B or $\mathrm{C}$ virus, cirrhosis, and genetic metabolic diseases (2). As regards treatment, surgical resection or local ablation is a good choice for non-cirrhotic patients with small tumors, while liver transplantation is a better choice for patients with early stages of HCC accompanied by decompensated cirrhosis (3-4). However, these treatments and availability of livers for transplantation are not applicable to the majority of patients. Therefore, it is necessary to further investigate the potential molecular mechanisms in HCC development in order to discover novel intervention targets for HCC.

S100A9 belongs to a family of 25 calcium-binding proteins and its gene is located on chromosome 1q21, a region that is unstable and rearrangeable in tumors (5-8). S100A9 often functions through binding with S100A8 which is another member of the same family, and has been linked to neoplastic disorders (9). It has been shown that S100A9 is upregulated and correlates with poor differentiation in $\mathrm{HCC}$ (10); however, at the same time, S100A9 upregulation has been found in the HCC cells of humans and mice and has been shown to protect Hep3B HCC cells from TNF- $\gamma$-induced apoptosis (11). A recent study demonstrated that extracellular S100A9 protein secreted from tumor cells plays a dual role, exhibiting anti-tumor or pro-tumor responses in a dose-dependent manner (12). At a concentration of $<20 \mu \mathrm{g} / \mathrm{ml}$, extracellular S100A9 has been shown to promote tumor cell proliferation in breast and prostate cancer; however, at a range of $25-250 \mu \mathrm{g} / \mathrm{ml}$, it has been shown to exert apoptotic effects on certain types of tumor cells(13-18). Nevertheless, the effect of extracellular S100A9 on HCC cells remains unclear.

Several molecular signaling pathways in HCC, particularly mitogen-activated protein kinase (MAPK) signaling, have been reported to play critical roles in carcinogenesis (19-21). Certain studies have demonstrated that S100A9 can enhance the activity of MAPK signaling in many types of cancer, including breast, prostate and colon cancer $(15,16,22)$. Whether 
S100A9 is involved in HCC development via the activation of the MAPK signaling pathway remains to be further elucidated.

In the present study, we aimed to investigate the effects of S100A9 on HepG2 HCC cells, as well as the molecular mechanisms underlying these effects. We found that S100A9 promoted the proliferation and invasion of HepG2 cells in vitro and tumor growth in vivo, and that the activation of the MAPK signaling pathway was involved in the S100A9-induced proliferation and invasion. These data indicate that S100A9 may play a role in HCC development.

\section{Materials and methods}

Cell culture and reagents. The HepG2 human HCC cell line and the L02 human normal liver cell line were kindly provided by Professor T.C. He (The University of Chicago Medical Center, Chicago, IL, USA). The HepG2 and L02 cells were maintained in Dulbecco's modified Eagle's medium (DMEM) supplemented with $10 \%$ fetal bovine serum (FBS; HyClone), $100 \mathrm{U} /$ $\mathrm{ml}$ of penicillin and $100 \mu \mathrm{g} / \mathrm{ml}$ of streptomycin. The cells were cultured in a humidified atmosphere of $5 \% \mathrm{CO}_{2}$ at $37^{\circ} \mathrm{C}$. The primary antibodies used in this study were as follows: mouse anti-hS100A9 monoclonal antibody (Cat no. 58706; Santa Cruz Biotechnology, Inc., Santa Cruz, CA, USA), rabbit anti-JNK monoclonal antibody (Cat no. 9253; Cell Signaling Technology, Danvers, MA, USA), rabbit anti-phosphor-JNK monoclonal antibody (Cat no. 4668; Cell Signaling Technology), rabbit anti-p38 monoclonal antibody (Cat no. 9212; Cell Signaling Technology), rabbit anti-phosphor-p38 monoclonal antibody (Cat no. 4511; Cell Signaling Technology), rabbit anti-ERK1/2 monoclonal antibody (Cat no. 4695; Cell Signaling Technology), rabbit anti-phosphor-ERK1/2 monoclonal antibody (Cat no. 3510; Cell Signaling Technology) and mouse anti- $\beta$-actin monclonal antibody (Cat no. 47778; Santa Cruz Biotechnology, Inc.). Specific inhibitors of p38 (SB203580) and ERK1/2 (PD98059) were obtained from Santa Cruz Biotechnology, Inc. and were used as per the manufacturer's instructions.

Recombinant protein preparation. The pGST-moluc-hS100A9 plasmid used in the current study has been described previously (23). Briefly, the pGST-moluc-hS100A9 was transformed into E. coli (BL21) by calcium chloride transformation. Isopropylthio- $\beta$-D-galactoside was used to induce the expression of GST-hS100A9 protein. The bacteria were then collected and sonicated on ice, and spun at $4^{\circ} \mathrm{C}$. The supernatant was incubated with glutathione-sepharose 4B beads, and GST-hS100A9 on the beads was eluted by elution buffer with reduced glutathione on ice. Finally the GST-hS100A9 protein was filtered and stored at $-80^{\circ} \mathrm{C}$. The control protein GST was prepared simultaneously (pGST-moluc plasmid).

Cell viability assay. Cell viability was measured using 3-(4,5-dimethylthiazol-2-yl)-2,5-diphenyltrazolium bromide (MTT) assay. A total of $2 \times 10^{3}$ cells were seeded into each well of 96-well culture plates, grown for $24 \mathrm{~h}$, then treated with GST or GST-S100A9 in DMEM containing 1\% FBS for 24, 48,72 , and $96 \mathrm{~h}$. After the indicated hours of incubation, $10 \mu \mathrm{l}$ of MTT reagent was added to the cells, followed by another $4 \mathrm{~h}$ of incubation at $37^{\circ} \mathrm{C}$. Dimethyl sulfoxide was added to dissolve the formazan product for $10 \mathrm{~min}$ at room temperature.
Finally the absorbance was measured at $492 \mathrm{~nm}$ using a microplate reader. Each condition was done in quintuplicate, and the experiment was repeated 3 times.

Colony forming assay. HepG2 cells during the log growth stage were seeded in 6 -well culture plates $\left(2 \times 10^{2}\right.$ cells/well) and treated with GST or GST-S100A9. After incubation for 2 weeks, the cells were stained with crystal violet and clones were counted. The colony-forming rate was obtained by the following calculation: (colony number/seeded cell number) $\mathrm{x} 100 \%$. The experiment was repeated 3 times.

Transwell invasion assay. The invasion assay was performed as previously described (24). The chamber of a nontype I-collagen-coated 24-well culture insert (Millipore, Billerica, MA, USA) was used, and the upper side of the insert was coated with ECM gel (Sigma, St. Louis, MO, USA). Briefly, HepG2 cells were placed in the upper chamber $\left(1 \times 10^{5}\right.$ cells) and incubated with GST or GST-S100A9 in serum-free medium, while the lower chamber contained medium only (600 $\mu \mathrm{l} /$ each insert) with 20\% FBS. After incubation for $24 \mathrm{~h}$, the transmembrane cells were dried, fixed with methanol, stained with hematoxylin and eosin (H\&E), and counted under a microscope at x100 magnification. The experiment was performed 3 times.

Tumorigenicity assays in nude mice. The in vivo experiments were performed in accordance with the guidelines established by the Animal Care and Use Committee, University Laboratory Animal Research. The 6-8-week old female nude mice were randomly divided into 3 groups ( $\mathrm{n}=5 /$ group). The HepG2 cells treated with GST- or GST-hS100A9 $\left(5 \times 10^{6}\right.$ cells/ cell line) for 3 days were suspended in $200 \mu 1$ phosphate buffer solution (PBS) and then injected subcutaneously into the posterior flank position of nude mice. The nude mice in the blank group were injected with untreated HepG2 cells $\left(5 \times 10^{6} /\right.$ mouse). Subcutaneous tumor growth was recorded every 5 days with vernier calipers. Tumor volume was calculated using the formula: $\pi / 6 \times\left(R_{\max } \times R_{\min }{ }^{2}\right)$, where $\mathrm{R}=$ tumor diameter. The mice were sacrificed after 50 days, and the tumor tissues were collected, fixed in buffered formaldehyde, embedded in paraffin, and sectioned for further histological and immunohistochemical analysis.

Western blot analysis. The cells treated with GST or GST-hS100A9 were harvested and lysed in radio immunoprecipitation assay (RIPA) buffer. The total cell lysate was centrifuged and the supernatant was denatured by boiling and loaded onto a $10 \%$ gradient SDS-PAGE. After SDS-PAGE, the proteins on the gel were blotted onto a PVDF membrane. The membrane was blocked with 5\% bovine serum albumin (BSA), and incubated with the primary antibodies and then with a secondary antibody conjugated with horseradish peroxidase. The proteins of interest were detected using the SuperSignal West Pico Chemiluminescent Substrate kit. The results were recorded using the Bio-Rad Electrophoresis Documentation (Gel Doc 1000) and Quantity One version 4.5.0 software (Bio-Rad, Hercules, CA, USA).

The primary antibodies used this study included mouse anti-S100A9 monoclonal antibody (1:1,000 dilution), rabbit 
anti-phosphor-p38 monoclonal antibody (1:1,000 dilution), rabbit anti-p38 monoclonal antibody (1:1,000 dilution), rabbit anti-phosphor-ERK1/2 monoclonal antibody (1:1,000 dilution), rabbit anti-ERK1/2 monoclonal antibody (1:1,000 dilution), rabbit anti-phosphor-JNK antibody (1:1,000 dilution), rabbit anti-JNK monoclonal antibody (1:1,000 dilution) and mouse anti- $\beta$-actin monoclonal antibody (1:1,000 dilution). The secondary antibodies included goat anti-rabbit IgG serum (1:5,000 dilution; Zhongshan Golden Bridge, Beijing, China) and goat anti-mouse IgG serum (1:5,000 dilution; Zhongshan Golden Bridge).

Immunohistochemistry (IHC). Paraffin-embedded subcutaneous tumor sections were processed for immunohistochemical analysis. Briefly, the deparaffinized and dehydrated sections were boiled for $10 \mathrm{~min}$ in $0.01 \mathrm{M}$ citrate buffer and incubated with $0.3 \%$ hydrogen peroxide $\left(\mathrm{H}_{2} \mathrm{O}_{2}\right)$ in methanol for $15 \mathrm{~min}$ to block endogenous peroxidase, then with primary and secondary antibodies tagged with the peroxidase in serial order. The desired brown reaction product was obtained after incubation with $0.05 \%$ 3,3-diaminobenzidine tetrachloride (DAB). Finally, the sections were counterstained with hematoxylin. The negative control groups treated as described above, except that the primary antibody was replaced by PBS.

Primary antibodies used in the assay were the mouse anti-S100A9 monoclonal antibody (1:300 dilution), rabbit antiphosphor-p38 monoclonal antibody (1:300 dilution) and rabbit anti-phosphor-ERK1/2 monoclonal antibody (1:300 dilution).

Inhibition of p38 and ERK1/2 with specific inhibitors and its effects on S100A9-induced proliferation and invasion of HepG2 cells. The cells were treated with $10 \mu \mathrm{M}$ SB203580 (p38 inhibitor) or $20 \mu \mathrm{M}$ PD98059 (ERK1/2 inhibitor) for $30 \mathrm{~min}$, followed by treatment with GST or GST-hS100A9 for the indicated periods of time. After treatment for $30 \mathrm{~min}$, the phosphorylation of p38 and ERK1/2 was detected by western blot analysis; after treatment for $24 \mathrm{~h}$, the cell invasion was detected by transwell invasion assay; after treatment for $72 \mathrm{~h}$, the cell proliferation was analyzed by MTT assay.

Statistical analysis. All values in the text and figures are presented as the means \pm standard error of mean (SEM). The differences were analyzed using one-way ANOVA followed by the Student-Newman-Keuls test, and all statistical analyses were performed using GraphPad Prism software. Statistical differences are presented at probability levels of $\mathrm{p}<0.05, \mathrm{p}<0.01$ and $\mathrm{p}<0.001$.

\section{Results}

Proliferative and invasive activities of HepG2 human HCC cells and S100A9 expression. In this study, we first used the L02 human normal liver cell line as a control to characterize the proliferation and invasive capability of the HepG2 human HCC cell line and to investigate the change in S100A9 expression in HepG2 cells by MTT assay, transwell invasion assay, western blot analysis and IHC. It was found that the absorbance of the formazan product by HepG2 cells at days 3 and 4 was $0.448 \pm 0.012$ and $0.524 \pm 0.017$ and the absorbance by $L 02$ cells was $0.403 \pm 0.01$ and $0.433 \pm 0.012(\mathrm{p}<0.01$ and p $<0.001$, respectively; Fig. 1A). At the same time, the transmembrane cell numbers were $81 \pm 4$ cells/field in the HepG2 cells and $24 \pm 3$ cells/field in the L02 cells ( $<<0.01$, Fig. 1B), suggesting that the HepG2 cells had strong proliferative and invasive capabilities. Western blot analysis showed a higher expression of S100A9 in the HepG2 cells compared with the L02 cells ( $p<0.05$, Fig. 1C). S100A9 upregulation in HepG2 cells was also confirmed by IHC and S100A9 was mainly localized in the cytoplasm (Fig. 1D).

S100A9-induced proliferation and invasion of HepG2 cells in vitro. To investigate the effects of extracellular S100A9 on the proliferation and invasion of HepG2 cells, we first prepared GST-hS100A9 and GST proteins, which were identified by SDS-PAGE and western blot analysis (Fig. 2A and B). Their purities were quantified by Quantity One software after SDS-PAGE and were found to be $>90 \%$ (Fig. 2A). The purified proteins were used to treat cells in our subsequent experiments.

The viability of HepG2 cells was assayed by MTT. After the HepG2 cells were treated with GST-hS100A9 at 0, 10, 20, 40,80 and $120 \mu \mathrm{g} / \mathrm{ml}$ for 4 successive days, we found that GST-hS100A9 at 10 and $20 \mu \mathrm{g} / \mathrm{ml}$ enhanced the cell proliferation at day $3(\mathrm{p}<0.05$ and $\mathrm{p}<0.01$, respectively) and day 4 $(\mathrm{p}<0.01$ and $\mathrm{p}<0.001$, respectively), and that GST-hS100A9 at $20 \mu \mathrm{g} / \mathrm{ml}$ had a more significant effect on cell proliferation ( $\mathrm{p}<0.001$ ), while the protein at 40,80 and $120 \mu \mathrm{g} / \mathrm{ml}$ had no effects on cell proliferation, compared with the GST group (Fig. 3A). thus, we selected $20 \mu \mathrm{g} / \mathrm{ml}$ as the concentration for all remaining experiments. Furthermore, after treatment with GST-hS100A9 for 2 weeks, we found a significant increase in colony formation in the GST-hS100A9 group and the colonyforming rate increased by $25.1 \%$ compared with that of the GST group (p<0.05) (Fig. 3B).

Cell invasiveness plays a crucial role in the process of tumor metastasis. Transwell invasion assay was used to detect the change in cellular invasion induced by GST-hS100A9. After treatment for $24 \mathrm{~h}$, the number of transmembrane cells in the GST-hS100A9 group increased by $43.1 \%$ compared with that of the GST group (p<0.01, Fig. 4).

S100A9 promotes tumorigenicity of HepG2 cells in vivo. Based on the promotive effect of exogenous S100A9 protein on the proliferation of HepG2 cells in vitro, we then sought to confirm this effect in vivo. The 3 groups of HepG2 cells (untreated or treated with GST or GST-hS100A9 for $72 \mathrm{~h}$ ) were subcutaneously implanted into nude mice. Tumor volume was measured every 5 days using a vernier caliper and the tumor tissues were surgically excised at day 50 after injection (Fig. 5A). At 3 weeks after injection, no tumors were found in the blank and GST groups, but were found in 4 out of 5 mice in the GST-hS100A9 group. Over the period of 50 days, the tumor volumes became palpable from day 25 to 50 and grew from $4 \pm 1.5$ to $71 \pm 27.8 \mathrm{~mm}^{3}$ in the blank group, from $5 \pm 1.0$ to $56 \pm 8.0 \mathrm{~mm}^{3}$ in the GST group, and from $60 \pm 45.0$ to $1,053 \pm 444.0 \mathrm{~mm}^{3}$ in the GST-hS100A9 group (Fig. 5B, Table I). The histological examination (H\&E staining) showed that the tumor cells in the 3 groups were obviously heterogeneous, with a large nucleus, a high nucleus/ cytoplasm ratio, with an irregular nuclear shape and variable nuclear size (Fig. 5C). 
A

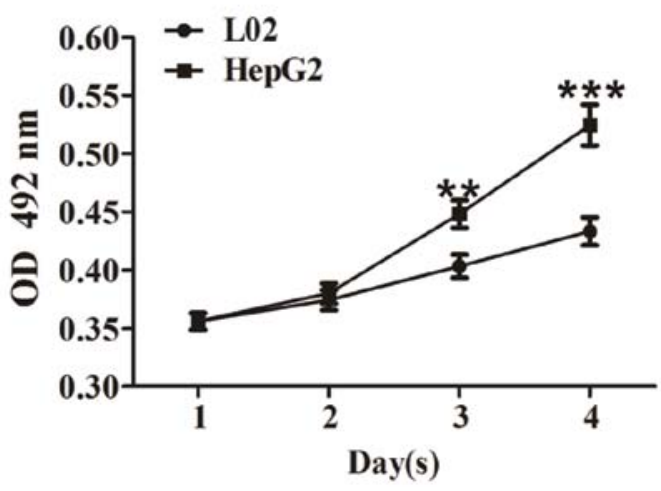

C

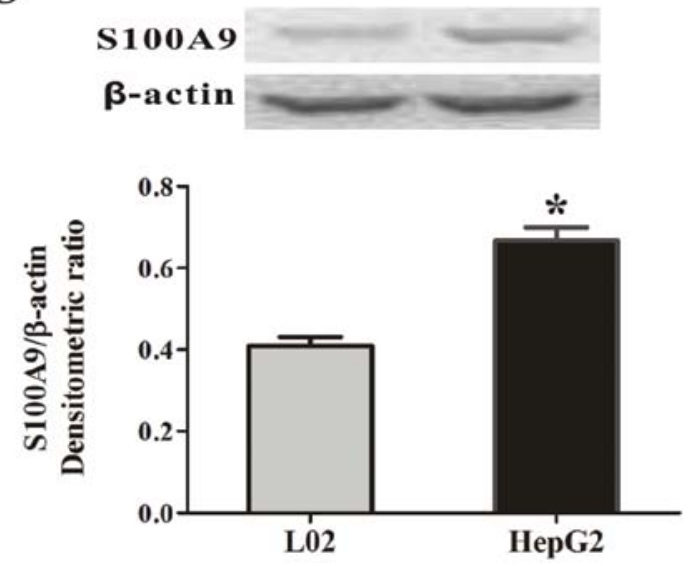

B
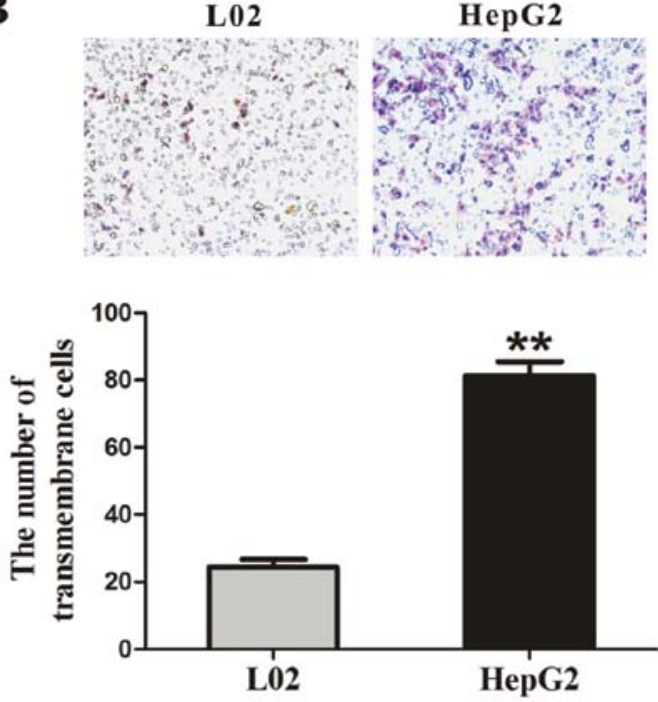

D

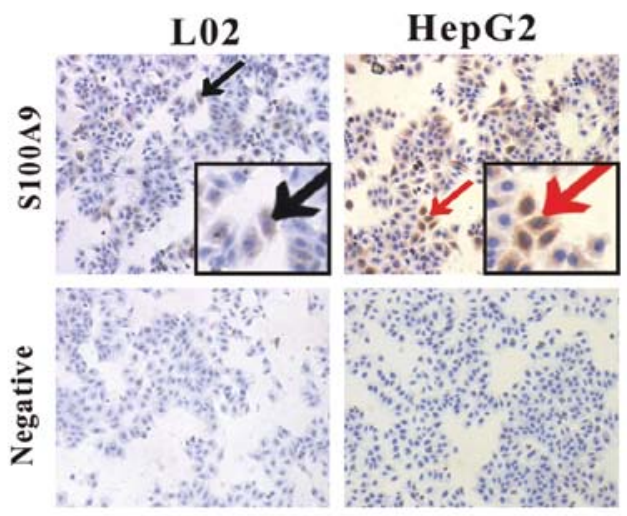

Figure 1. Characterization of the HepG2 human hepatocellular carcinoma cell line. (A) MTT assay. The viability of the HepG2 human hepatocellular carcinoma cells and the L02 human normal liver cells was detected by MTT assay at 24, 48, 72 and $96 \mathrm{~h}$, as described in Materials and methods. The absorbance was measured at $492 \mathrm{~nm}$ using a microplate reader. The results represent the mean absorbance \pm SEM of 3 independent experiments. HepG2 cells had a strong proliferative ability. ${ }^{* *} \mathrm{p}<0.01$ or ${ }^{* * * *} \mathrm{p}<0.001$ vs. L02. (B) Transwell invasion assay. Equal numbers of HepG2 cells and L02 cells were subjected to cell invasion using transwell invasion assay. After $24 \mathrm{~h}$, the transmembrane cells were stained with hematoxylin and eosin (H\&E) and counted under a microscope. Representative images of transmembrane cells are shown in the upper panel, and the mean number of transmembrane cells \pm SEM per microscopic field of 3 independent experiments are quantified in the lower panel. Magnification, $x 100 .{ }^{* *} p<0.01$ vs. L02. (C) S100A9 expression in HepG2 and L02 cells was measured by western blot analysis. The densitometric ratios were normalized to those of $\beta$-actin, and the results are expressed as the mean densitometric ratios \pm SEM in the lower panel. "p $<0.05$ vs. L02. (D) IHC assay of S100A9 expression in HepG2 and L02 cells. Representative images are shown. Black arrows, S100A9-expressing L02 cells (brown); red arrows, S100A9-expressing HepG2 cells (brown). Magnification, x100. Inlets represent higher magnification.

A

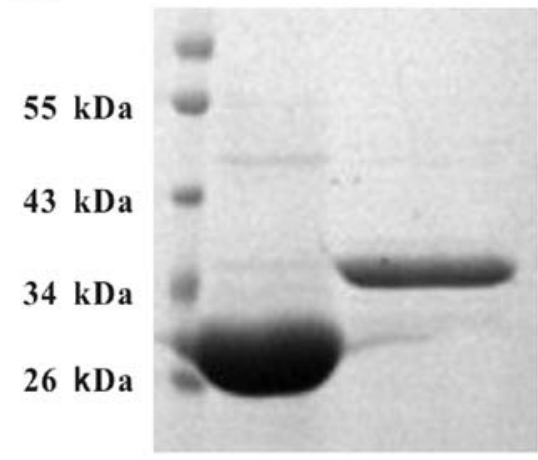

B

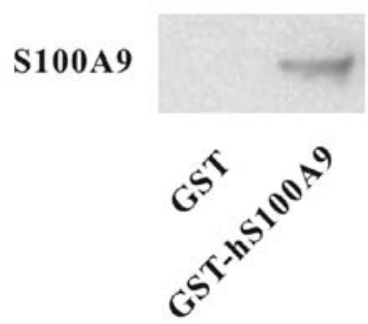

Figure 2. Identification of GST-hS100A9 by SDS-PAGE and western blot analysis. (A) Recombinant protein GST-hS100A9 was approximately $40 \mathrm{kDa}$, and GST was approximately $26 \mathrm{kDa}$; their purities were $>90 \%$ (by Quantity One software). (B) GST-hS100A9 was recognized by anti-S100A9 antibody by western blot analysis. Left lane, GST protein; right lane, GST-hS100A9 protein. 
A

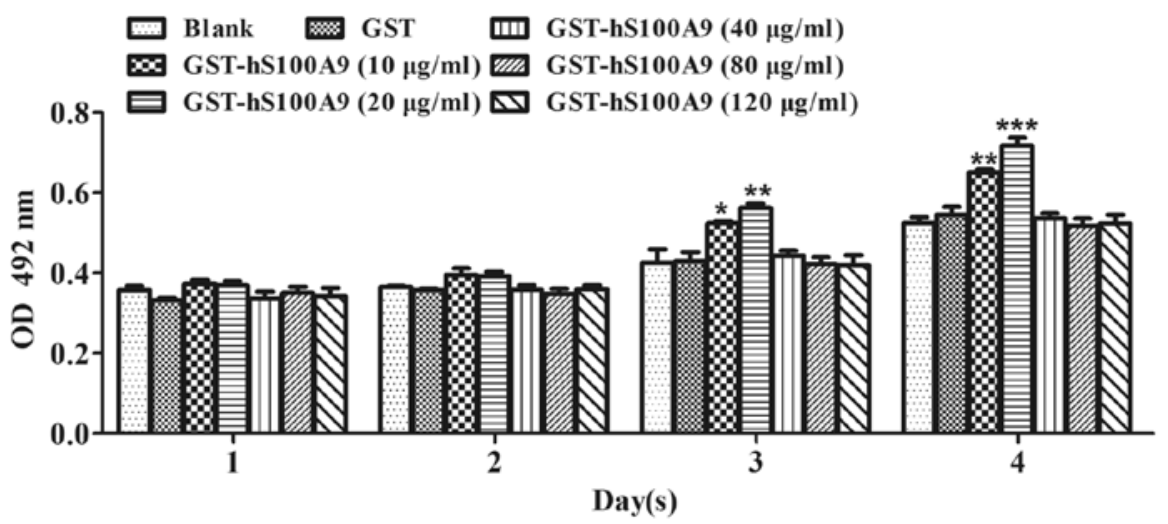

B
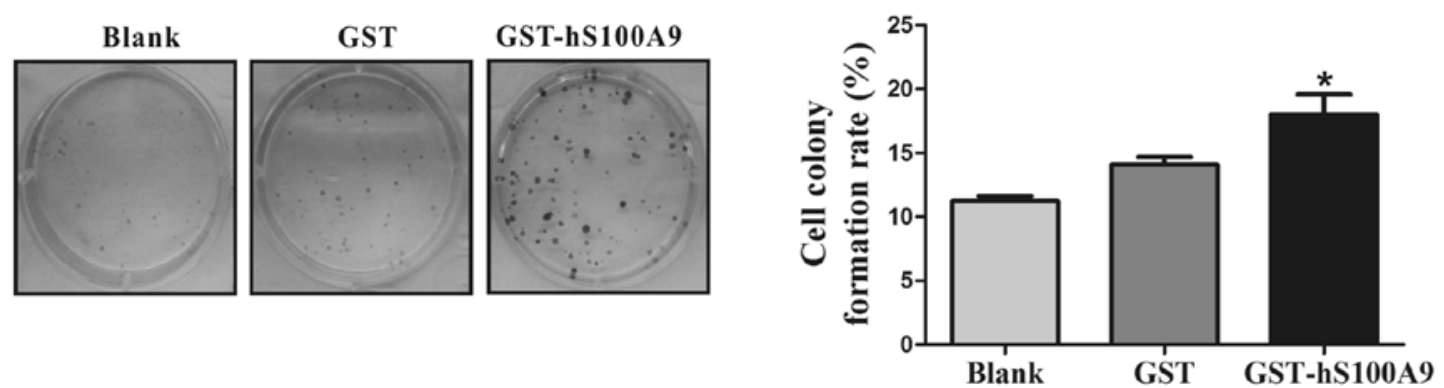

Figure 3. Exogenous S100A9 promotes the proliferation of HepG2 cells. (A) MTT assay. HepG2 cells $\left(2 \times 10^{3}\right.$ cells/each well) were seeded in 96-well plates, and treated with GST or GST-hS100A9 for 24, 48, 72 and $96 \mathrm{~h}$ before the absorbance was measured at $492 \mathrm{~nm}$ using a microplate reader. The results represent the mean absorbance \pm SEM of 3 independent experiments. $p<0.05,{ }^{* *} p<0.01$ and ${ }^{* * * *} p<0.001$, GST-hS100A9 vs. GST control group. (B) Colony forming assay of the HepG2 cells treated with GST-hS100A9 $(20 \mu \mathrm{g} / \mathrm{ml})$. The number of colonies increased significantly in the GST-hS100A9 group. Representative images of the colony-forming unit are presented in the left panel, and colony-forming rates for each group are quantified in the right panel. The colony-forming rate was obtained by the following calculatino: (colony number/seeded cell number) x100\%. The experiment was repeated 3 times. "p<0.05, GST-hS100A9 vs. GST control.
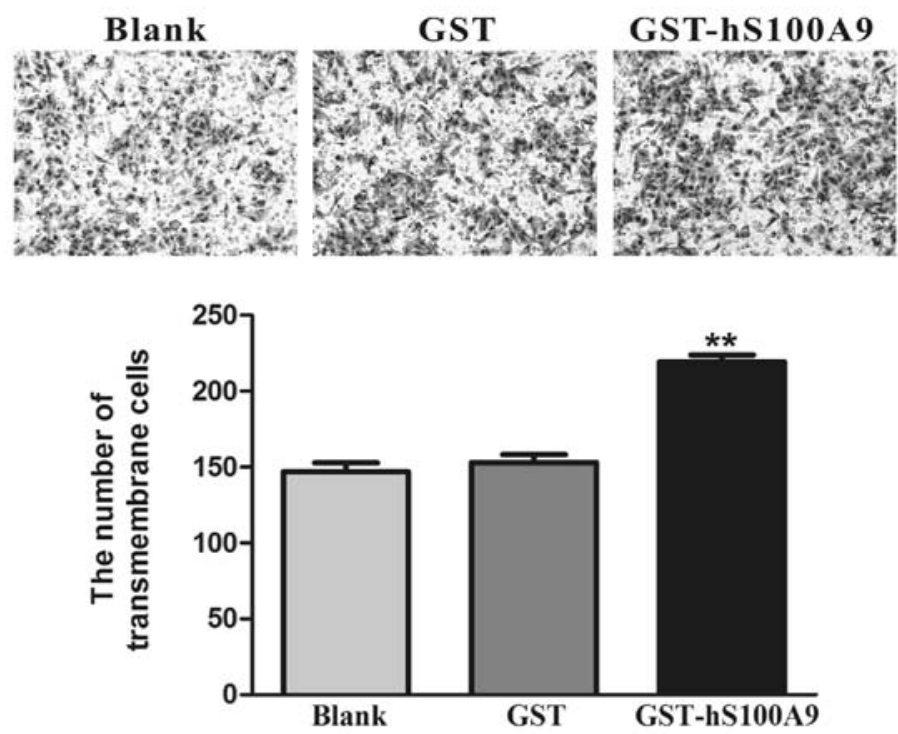

Figure 4. Exogenous S100A9 enhances the invasive ability of the HepG2 cells. HepG2 cells were either left untreated or treated with GST (20 $\mu \mathrm{g} / \mathrm{ml}$ ) or GST$\mathrm{hS100A9}(20 \mu \mathrm{g} / \mathrm{ml})$ for $24 \mathrm{~h}$ for transwell invasion assay. The transmembrane cells were stained with H\&E and counted under a microscope. Representative images of transmembrane cells are shown in the upper panel; the mean number of transmembrane cells \pm SEM per microscopic field of 3 independent experiments is quantified in the lower panel. Magnification, x100. "p<0.01, GST-hS100A9 vs. GST control.

S100A9-induced activation of the MAPK signaling pathway in HepG2 cells. It has previously been reported that S100A9 activates the MAPK signaling pathway $(15-16,22)$. To deter- mine whether S100A9 is involved in the activation of the MAPK signaling pathway in HepG2 cells, we detected and analyzed the phosphorylation of MAPKs in cell lysates of 
A

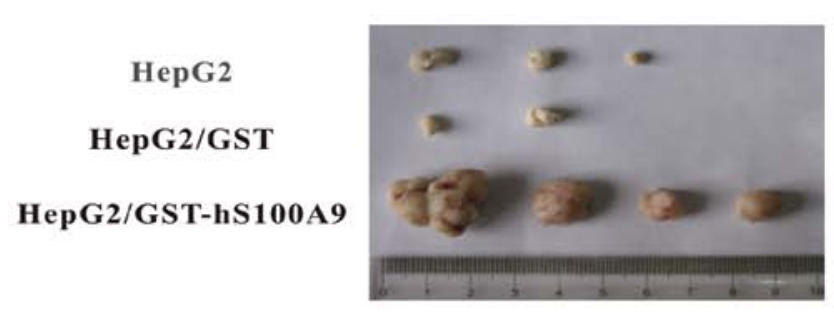

B

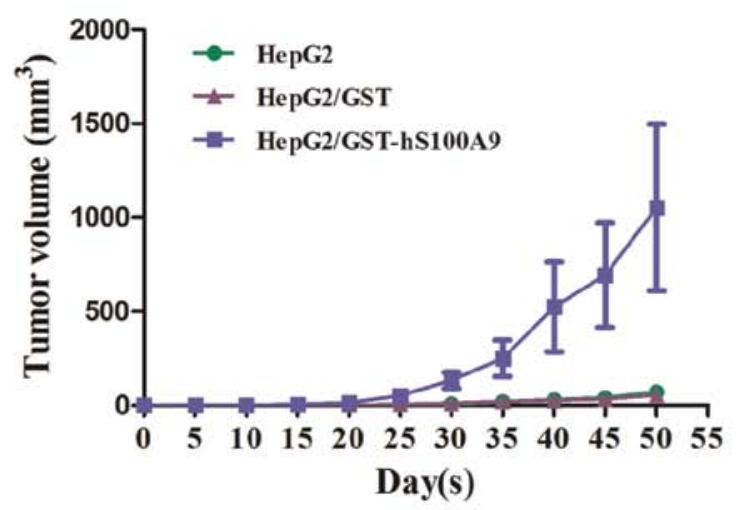

C
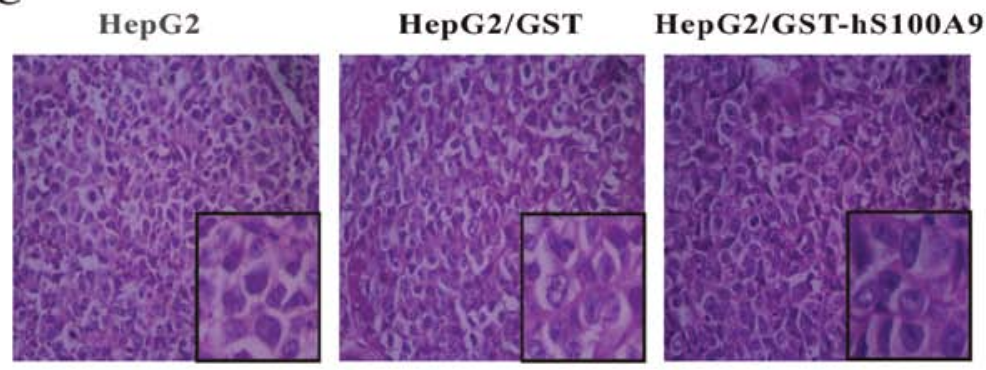

Figure 5. Exogenous S100A9 promotes tumor formation in vivo. (A) Excised tumor tissues from all the groups. (B) Tumor growth curves of all groups (n=5/ group). The cells were pre-treated with GST or GST-hS100A9 and were then subcutaneously implanted into nude mice. Tumor volume was measured every 5 days using a vernier caliper. S100A9 promoted the growth of HepG2 cells in vivo. (C) H\&E staining of tumor tissues. All tumors showed a large nucleus, a high nucleus/cytoplasm ratio, an irregular nuclear shape and variable nuclear size, while no variation in heterogeneity was found among these groups. Magnification, $\mathrm{x} 400$.

Table I. Tumorigenicity of the 3 groups of HepG2 cells subcutaneously implanted into nude mice.

\begin{tabular}{lccc}
\hline Group & $\begin{array}{c}\text { No. }(\%) \text { of mice } \\
\text { with tumors }\end{array}$ & $\begin{array}{c}\text { Tumor onset } \\
\text { (day) }\end{array}$ & $\begin{array}{c}\text { Average tumor } \\
\text { volume }\left(\mathrm{mm}^{3}\right)\end{array}$ \\
\hline HepG2 & $3(60)$ & $28.7 \pm 1.7$ & $71 \pm 27.8$ \\
HepG2/GST & $2(40)$ & $29.5 \pm 2.5$ & $56 \pm 8.0$ \\
HepG2/GST-S100A9 & $4(80)$ & $17.0 \pm 0.7$ & $1,053 \pm 444.0$
\end{tabular}

There were 5 mice in each group. HepG2/GST and HepG2/GST-S100A9 indicate the nude mice which were subcutaneously injected with GSTand GST-S100A9-treated HepG2 cells, respectively. The experiments were repeated twice with similar results.

HepG2 cells treated with GST-hS100A9 for different periods of time by western blot analysis. The results showed that GST-hS100A9 had no effect on the phosphorylation of SAPK/ JNK but enhanced the phosphorylation of ERK1/2 (within 15 min, $\mathrm{p}<0.01$ ) and $\mathrm{p} 38$ (within $30 \mathrm{~min}, \mathrm{p}<0.01$ ) MAPKs within 60 min (Fig. 6A). Furthermore, the role of S100A9 in the activation of $\mathrm{p} 38$ and ERK1/2 was also confirmed in vivo. IHC showed that there was a higher expression of S100A9, phosphor-p38 and phosphor-ERK1/2 in the subcutaneous tumor tissues in the GST-hS100A9 group, compared with the other 2 groups (Fig. 6B). These data demonstrate that S100A9 enhances the activity of the MAPK signaling pathway (p38 and ERK1/2) in HepG2 cells.

Impact of the inhibition of MAPK signaling on S100A9induced proliferation and invasion of HepG2 cells. We further investigated whether the activation of the MAPK signaling pathway is involved in the S100A9-induced proliferation and invasion of HepG2 cells. The specific inhibitors of $\mathrm{p} 38$ (SB203580) and ERK1/2 (PD98059) were used to pre-treat the HepG2 cells and the S100A9-induced phosphorylation of p38 and ERK $1 / 2$ was reversed by SB203580 ( $\mathrm{p}<0.01)$ and PD98059 $(\mathrm{p}<0.01)$, respectively (Fig. 7A and B). At the same time, we detected changes in cell proliferation and invasion in the presence and absence of SB203580 or PD98059. We found that the S100A9-induced proliferation of HepG2 cells was reversed by PD98059 (p<0.05), but not by SB203580 (Fig. 7C) and that the S100A9-induced cell invasion of HepG2 cells was reversed by SB203580 ( $<<0.05$ ), but not reversed by PD98059 (Fig. 7D). These data suggest that the promotive role of S100A9 in the proliferation and invasion of HepG2 cells may be mediated by the phosphorylation of p38 and ERK1/2, respectively.

\section{Discussion}

As mentioned in the Introduction, it is necessary to elucidate the potential molecular mechanisms involved in HCC develop- 
A

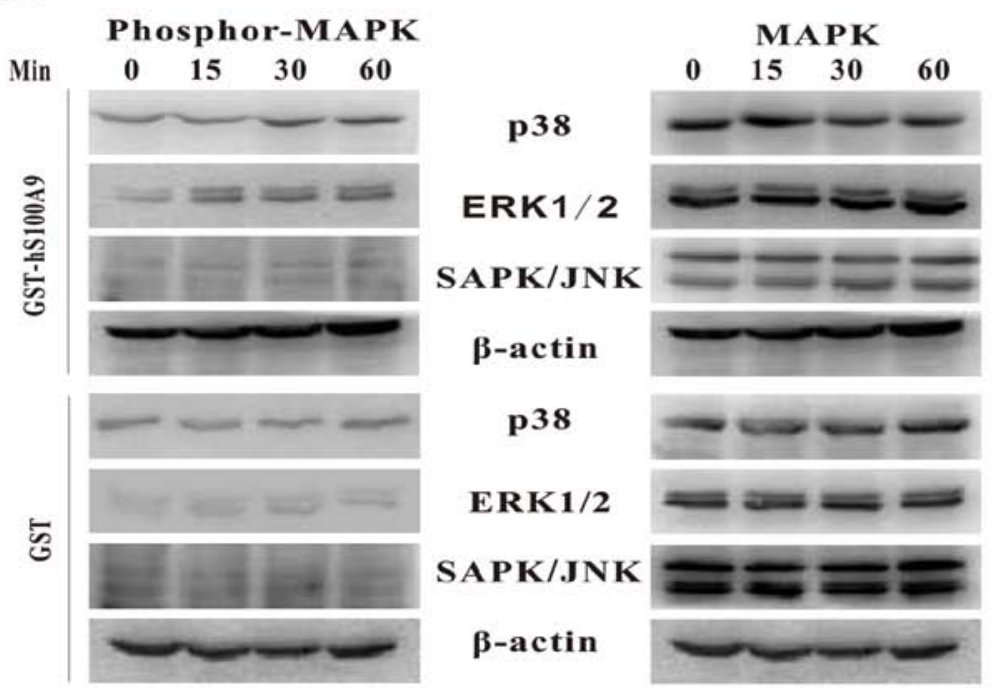

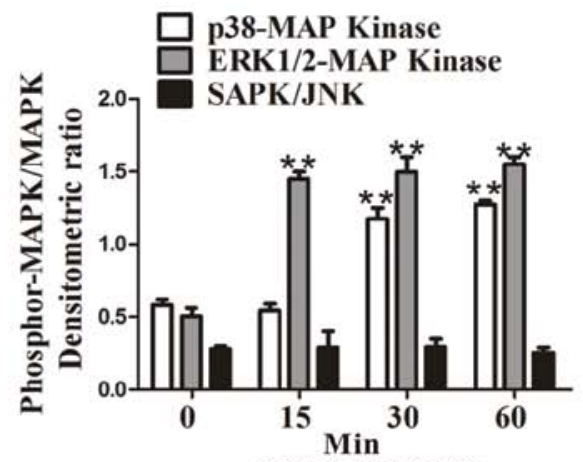

(GST-hS100A9)

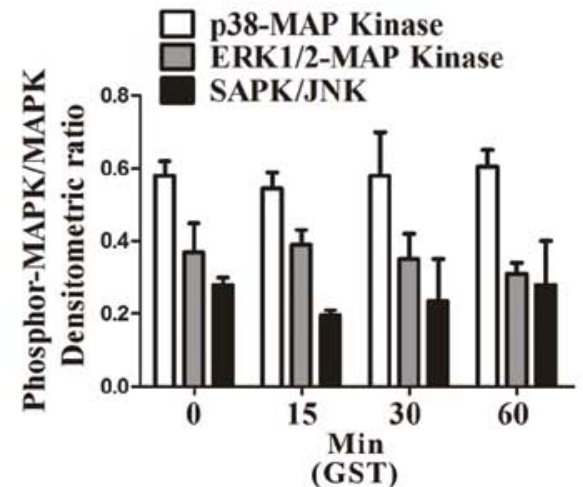

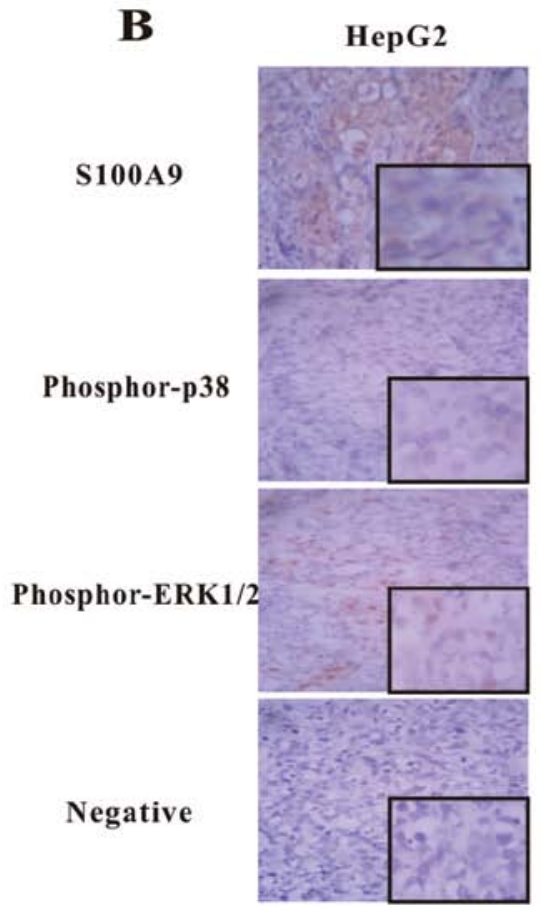

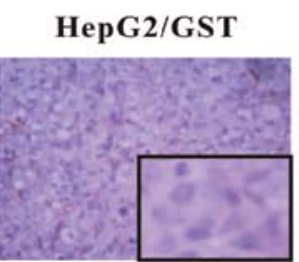

HepG2/GST-hS100A9
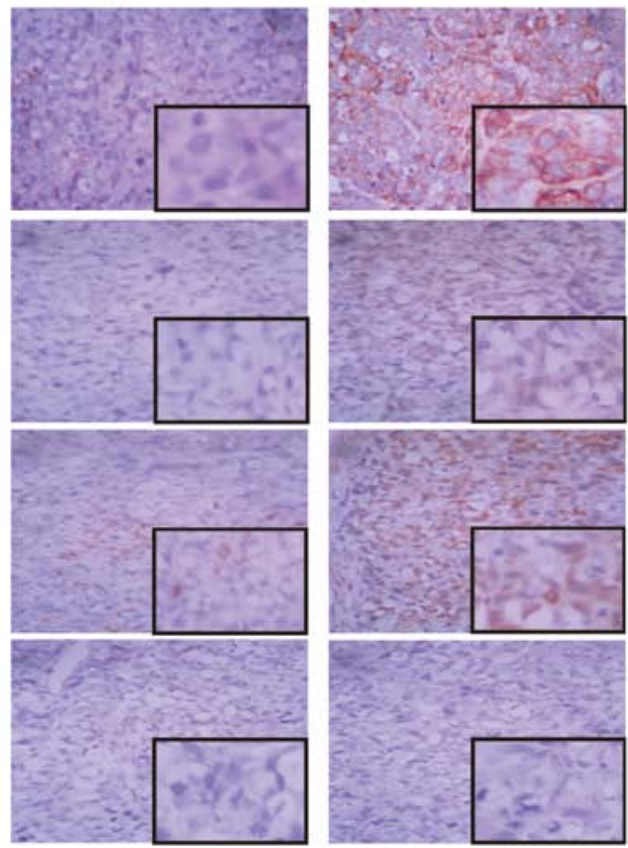

Figure 6. Exogenous S100A9 induces the activation of the MAPK signaling pathway (p38 and ERK1/2) in HepG2 cells. (A) HepG2 cells were stimulated with GST $(20 \mu \mathrm{g} / \mathrm{ml})$ and GST-S100A9 $(20 \mu \mathrm{g} / \mathrm{ml})$ for $0,15,30$ and $60 \mathrm{~min}$, and cell lysates were analyzed by western blot analysis using respective antibodies against phosphorylated MAPKs. Total p38, ERK1/2, SAPK/JNK and $\beta$-actin were included as the loading controls. The densitometric ratios were compared to the controls and then normalized to the $\beta$-actin control, and are shown in the upper right panel. ${ }^{* *}$ p $<0.01$, GST-hS100A9 (15, 30 or 60 min) vs. GST-hS100A9 (0 min). (B) Tumor tissues derived from mice in the 3 groups (injected with HepG2 cells, GST-treated HepG2 cells and GST-hS100A9-treated HepG2 cells) were subjected to immunohistochemical staining for S100A9, phosphorylated p38 and ERK1/2. An intense staining for S100A9, phosphorylated p38 and ERK1/2 was shown in the GST-hS100A9 group compared with the other 2 control groups. Representative images are shown. Magnification, $\mathrm{x} 400$. Inlets represent higher magnification.

ment in order to discover novel therapeutic strategies against HCC. S100A9 has been shown to play a role in various types of tumor $(14-16,22)$. A previous study demonstrated that
S100A9 expression was associated with tumor differentiation and vascular invasion in HCC (10). Nonetheless, the exact role of S100A9 in the progression of HCC has not been extensively 
A
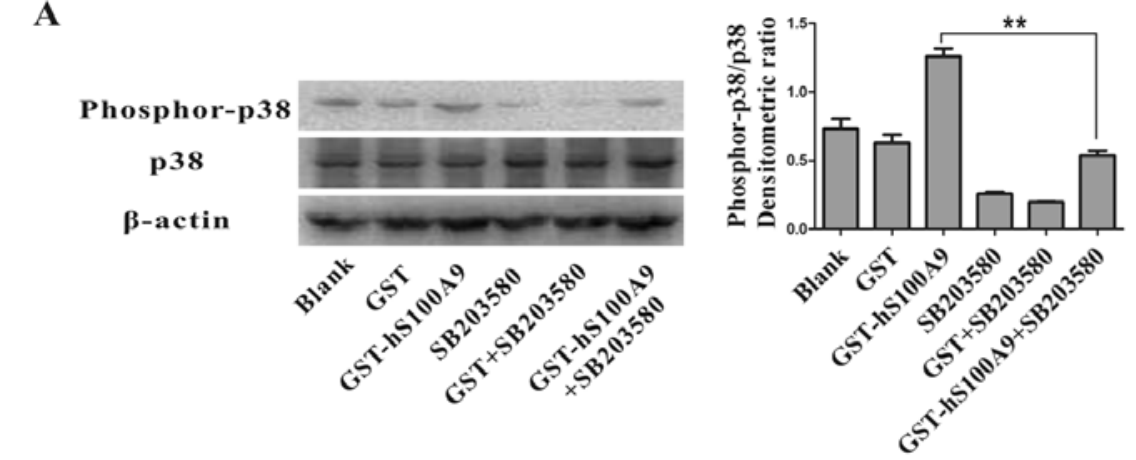

$\mathbf{B}$
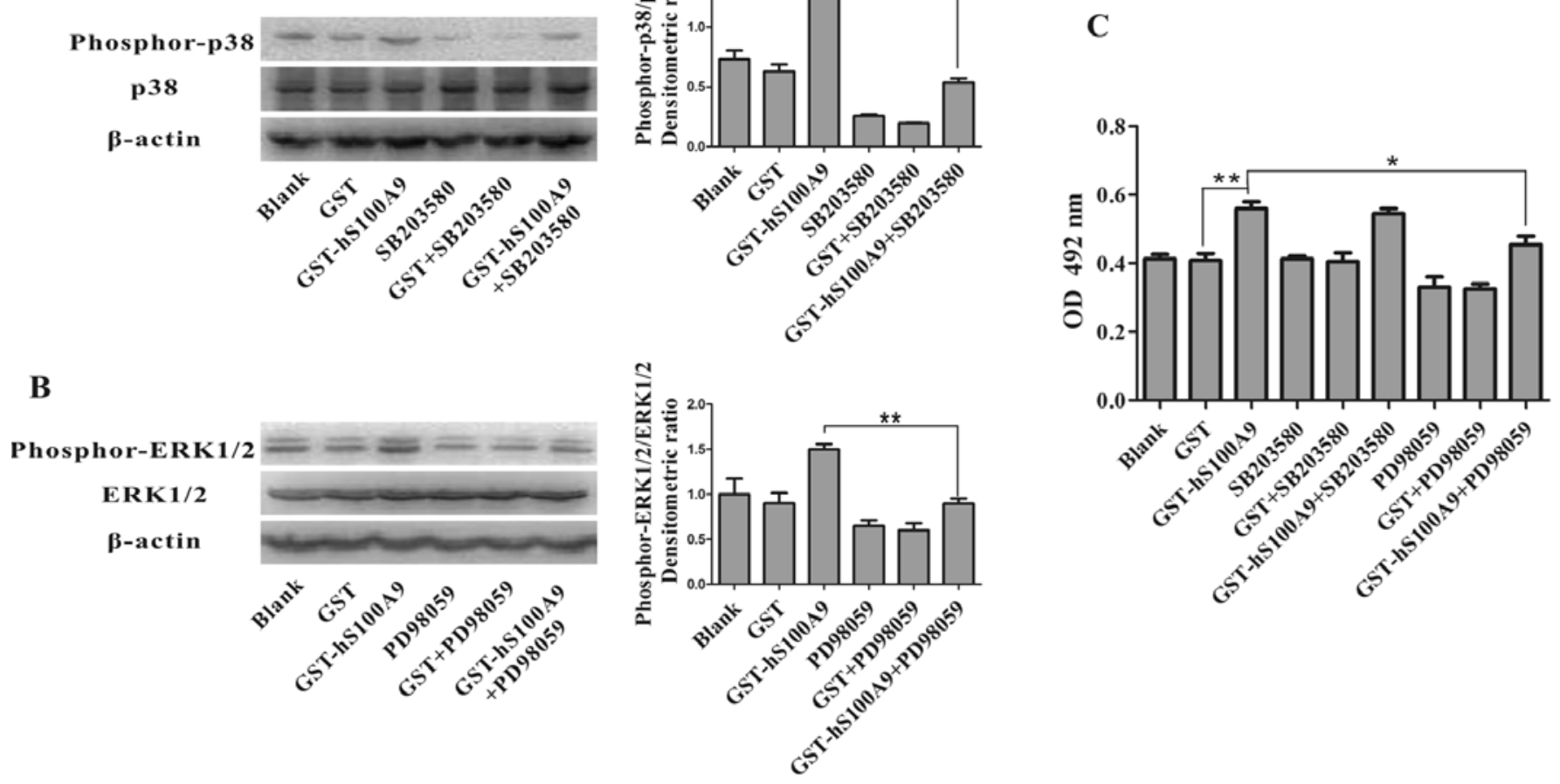

D
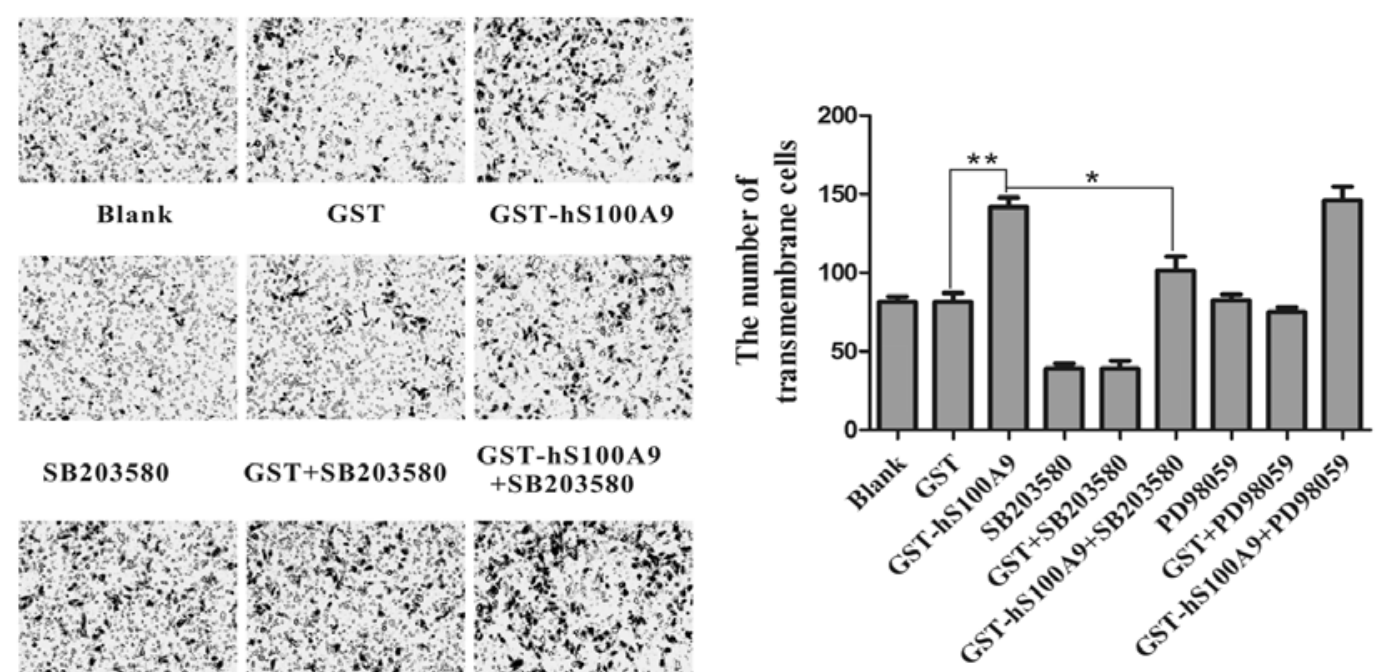

Figure 7. The promotive role of S100A9 in the proliferation and invasion of HepG2 cells. The proliferation and invasion was reversed by inhibitors of ERK1/2 (PD98059) and p38 (SB203580), respectively. (A and B) HepG2 cells were pre-treated with the inhibitors of p38 (SB203580, $10 \mu \mathrm{M}$ ) and ERK1/2 (PD98059, $20 \mu \mathrm{M})$ for a period of $30 \mathrm{~min}$ prior to treatment for $30 \mathrm{~min}$ with GST $(20 \mu \mathrm{g} / \mathrm{ml})$ or GST-hS100A9 $(20 \mu \mathrm{g} / \mathrm{ml})$. The cell lysates were then analyzed by western blot analysis using respective antibodies against phosphorylated p38 or ERK1/2. The densitometric ratios were compared to the control and then normalized to the $\beta$-actin control. The promotive role of S100A9 in the phosphorylation of (A) p38 and (B) ERK1/2. The phosphorylation of p38 and ERK1/2 was reversed by SB203580 and PD98059, respectively. ${ }^{* *}$ p $<0.01$, GST-hS100A9 vs. GST-hS100A9+SB203580 or GST-hS100A9+PD98059. (C) HepG2 cells were treated with GST-S100A9 $(20 \mu \mathrm{g} / \mathrm{ml})$ in the presence of SB203580 or PD98059 for $72 \mathrm{~h}$. Cell viability was measured by MTT assay. The promotive role of S100A9 in cell proliferation was reversed by PD98059. The results represent the mean absorbance \pm SEM of 3 independent experiments. ${ }^{* *}<<0.01$, GST-hS100A9 vs. GST control; " $p<0.01$, GST-hS100A9 vs. GST-hS100A9 + PD98059. (D) HepG2 cells were treated with GST-S100A9 (20 $\mu \mathrm{g} / \mathrm{ml})$ in the presence of SB203580 or PD98059 for $24 \mathrm{~h}$. Cell invasion was measured by transwell invasion assay. The transmembrane cells were stained with H\&E and were counted under a microscope. The results weere obtained from 5 randomly selected fields for each well, and representative images of transmembrane cells are shown in the left panel; the mean number of transmembrane cells \pm SEM per microscopic field of 3 independent experiments are quantified in the right panel. Magnification, $\mathrm{x} 100 .{ }^{* *} \mathrm{p}<0.01$, GST-hS100A9 vs. GST control; ${ }^{*} \mathrm{p}<0.05$, GST-hS100A9 vs. GST-hS100A9+SB203580.

studied. Therefore, in this study, we investigated the effects of S100A9 protein on the cell proliferation and invasion of HepG2 HCC cells and the possible underlying mechanisms.
Our results showed that S100A9 was upregulated in the HepG2 HCC cells compared with the L02 normal liver cells. These results are consistent with those from previous studies 
demonstrating that S100A9 is upregulated in other HCC cell lines, including Hep3B and HuH-7 cells (11). A number of studies have demonstrated that extracellular S100A9 secreted from the tumor cells functions as a danger signal by activating signaling cascades and promoting tumor cell proliferation in breast, colon and pancreatic cancer (14-16). However, little is known about the effect of extracellular S100A9 on HCC cells. Our data showed that exogenous S100A9 protein promoted the proliferation and growth of HepG $2 \mathrm{HCC}$ cells in vitro and in vivo, indicating that S100A9 is involved in HCC development. We also found that treatment with S100A9 resulted in an increase in cell invasion, suggesting that S100A9 may be involved in the metastasis of HCC cells. These results are consistent with those from previous reports, demonstrating that S100A9 plays a role in the metastasis of prostate, colorectal and breast cancer $(16,25-26)$. Of note, our results showed that higher concentrations of S100A9 (40-120 $\mu \mathrm{g} / \mathrm{ml})$ had no apoptotic effects on HepG2 cells, which vary from the results of previous reports demonstrating that S100A9 at the range of $25-250 \mu \mathrm{g} / \mathrm{ml}$ exerted apoptotic effects on certain types of tumor cells (13-18). This discrepancy suggests the apoptotic effects exerted by S100A9 are dependent on the tumor cell type.

Activated MAPK signaling plays a central role in HCC development (27-31), providing us with a novel target for intervention in $\mathrm{HCC}$ treatment. A number of previous studies have shown that extracellular S100A9 activates the MAPK signaling pathway in a variety of cell lines, mostly through the receptor for advanced glycation end-products (RAGE) (14-16,32-34). Therefore, we investigated the effect of S100A9 on MAPK signaling in HCC and found that S100A9 enhanced the phosphorylation of p38 and ERK1/2 MAPKs in HepG2 cells in vitro. This phosphorylation of p38 and ERK1/2 was inhibited by the specific inhibitors, SB203580 and PD98059, respectively; this effect was also demonstrated in vivo. These data suggest that S100A9 is involved in HCC development by the activation of the MAPK signaling pathway. A previous study demonstrated that the expression of RAGE mRNA was higher in HCC compared to normal liver tissues (35), and that the binding of RAGE with its ligands activated the MAPK signaling pathway (36). However, further studies are required to elucidate whether the S100A9-enhanced phosphorylation of p38 and ERK1/2 MAPKs in HepG2 cells is mediated by RAGE.

The results from the present study demonstrated that the S100A9-induced cell proliferation was reversed by PD98059 (an ERK1/2 inhibitor), suggesting that S100A9 promotes the proliferation of HepG 2 cells via the ERK $1 / 2$ signal transduction pathway. These results are consistent with those from previous studies, demonstrating that the ERK1/2 signal transduction pathway plays a crucial role in liver tumor cell proliferation and tumorigenesis $(29,37)$. At the same time, we found that the S100A9-induced cell invasion was reversed by SB203580 (a p38 inhibitor), which suggests that S100A9 promotes the invasion of HepG2 cells via the p38 signal transduction pathway. These results are supported by previous studies showing that the p38 signal transduction pathway plays an important role in the upregulation of the expression of matrix metalloproteinases (MMPs), correlating with an increased invasive phenotype of tumor cells (38-41).
In conclusion, the current observations indicate that S100A9 is upregulated in the HepG2 HCC cell line, and that exogenous S100A9 promotes the proliferation and invasion of HepG2 HCC cells by activating ERK1/2 and p38 MAPKs, respectively. Therefore, S100A9 may be a novel intervention target for HCC treatment.

\section{Acknowledgements}

The authors would like to thank Professor T.C. He (The University of Chicago, Medical center) for his kind provision of the HepG2 and L02 cell lines. The present study was supported by the National Natural Science Foundation of China (grant no. 30772548) and the Foundation for Excellent Master Dissertation of College of Laboratory Medicine at Chongqing Medical University.

\section{References}

1. Jemal A, Bray F, Center MM, Ferlay J, Ward E and Forman D: Global cancer statistics. CA Cancer J Clin 61: 69-90, 2011.

2. Cornella H, Alsinet $C$ and Villanueva A: Molecular pathogenesis of hepatocellular carcinoma. Alcohol Clin Exp Res 35: 821-825, 2011.

3. Bruix $\mathbf{J}$ and Sherman M: Management of hepatocellular carcinoma. Hepatology 42: 1208-1236, 2005.

4. Rossi L, Zoratto F, Papa A, et al: Current approach in the treatment of hepatocellular carcinoma. World J Gastrointest Oncol 2: 348-359, 2010.

5. Donato R: S100: a multigenic family of calcium-modulated proteins of the EF-hand type with intracellular and extracellular functional roles. Int J Biochem Cell Biol 33: 637-668, 2001.

6. Heizmann CW, Fritz G and Schafer BW: S100 proteins: structure, functions and pathology. Front Biosci 7: d1356-d1368, 2002.

7. Donato R: Intracellular and extracellular roles of S100 proteins. Microsc Res Tech 60: 540-551, 2003.

8. Marenholz I, Heizmann CW and Fritz G: S100 proteins in mouse and man: from evolution to function and pathology (including an update of the nomenclature). Biochem Biophys Res Commun 322: 1111-1122, 2004

9. Benedyk M, Sopalla C, Nacken W, et al: HaCaT keratinocytes overexpressing the S100 proteins S100A8 and S100A9 show increased NADPH oxidase and NF-kappaB activities. J Invest Dermatol 127: 2001-2011, 2007.

10. Arai K, Yamada T and Nozawa R: Immunohistochemical investigation of migration inhibitory factor-related protein (MRP)-14 expression in hepatocellular carcinoma. Med Oncol 17: 183-188, 2000.

11. Nemeth J, Stein I, Haag D, et al: S100A8 and S100A9 are novel nuclear factor kappa $\mathrm{B}$ target genes during malignant progression of murine and human liver carcinogenesis. Hepatology 50: $1251-1262,2009$.

12. Srikrishna G: S100A8 and S100A9: new insights into their roles in malignancy. J Innate Immun 4: 31-40, 2012.

13. Ghavami S, Chitayat S, Hashemi M, et al: S100A8/A9: a Janus-faced molecule in cancer therapy and tumorgenesis. Eur J Pharmacol 625: 73-83, 2009.

14. Turovskaya O, Foell D, Sinha P, et al: RAGE, carboxylated glycans and S100A8/A9 play essential roles in colitis-associated carcinogenesis. Carcinogenesis 29: 2035-2043, 2008.

15. Ghavami S, Rashedi I, Dattilo BM, et al: S100A8/A9 at low concentration promotes tumor cell growth via RAGE ligation and MAP kinase-dependent pathway. J Leukoc Biol 83: 1484-1492, 2008.

16. Hermani A, De Servi B, Medunjanin S, Tessier PA and Mayer D: S100A8 and S100A9 activate MAP kinase and NF-kappaB signaling pathways and trigger translocation of RAGE in human prostate cancer cells. Exp Cell Res 312: 184-197, 2006.

17. Ghavami S, Kerkhoff C, Chazin WJ, et al: S100A8/9 induces cell death via a novel, RAGE-independent pathway that involves selective release of Smac/DIABLO and Omi/HtrA2. Biochim Biophys Acta 1783: 297-311, 2008.

18. Ghavami S, Eshragi M, Ande SR, et al: S100A8/A9 induces autophagy and apoptosis via ROS-mediated cross-talk between mitochondria and lysosomes that involves BNIP3. Cell Res 20: 314-331, 2010. 
19. Schmidt CM, McKillop IH, Cahill PA and Sitzmann JV: The role of cAMP-MAPK signalling in the regulation of human hepatocellular carcinoma growth in vitro. Eur J Gastroenterol Hepatol 11: 1393-1399, 1999.

20. Huynh H, Nguyen TT, Chow KH, Tan PH, Soo KC and Tran E: Over-expression of the mitogen-activated protein kinase (MAPK) kinase (MEK)-MAPK in hepatocellular carcinoma: its role in tumor progression and apoptosis. BMC Gastroenterol 3: 19, 2003.

21. Nakagawa $\mathrm{H}$ and Maeda S: Molecular mechanisms of liver injury and hepatocarcinogenesis: focusing on the role of stress-activated MAPK. Patholog Res Int 2012: 172894, 2012.

22. Ichikawa M, Williams R, Wang L, Vogl T and Srikrishna G: S100A8/A9 activate key genes and pathways in colon tumor progression. Mol Cancer Res 9: 133-148, 2011.

23. You L, Xu LL, Guo YY, et al: Prokaryotic expression, purification and identification of GST-human S100A9 fusion protein. Chin J Biochem Pharm 32: 253-256, 2011

24. Punathil T, Tollefsbol TO and Katiyar SK: EGCG inhibits mammary cancer cell migration through inhibition of nitric oxide synthase and guanylate cyclase. Biochem Biophys Res Commun 375: 162-167, 2008.

25. Ang CW, Nedjadi T, Sheikh AA, et al: Smad4 loss is associated with fewer S100A8-positive monocytes in colorectal tumors and attenuated response to S100A8 in colorectal and pancreatic cancer cells. Carcinogenesis 31: 1541-1551, 2010.

26. Arai K, Takano S, Teratani T, Ito Y, Yamada T and Nozawa R: S100A8 and S100A9 overexpression is associated with poor pathological parameters in invasive ductal carcinoma of the breast. Curr Cancer Drug Targets 8: 243-252, 2008.

27. Sakurai T,He G,Matsuzawa A, et al: Hepatocyte necrosis induced by oxidative stress and IL-1 alpha release mediate carcinogeninduced compensatory proliferation and liver tumorigenesis. Cancer Cell 14: 156-165, 2008.

28. Spaziani A, Alisi A, Sanna D and Balsano C: Role of p38 MAPK and RNA-dependent protein kinase (PKR) in hepatitis $C$ virus core-dependent nuclear delocalization of cyclin B1. J Biol Chem 281: 10983-10989, 2006.

29. Gailhouste L, Ezan F, Bessard A, et al: RNAi-mediated MEK1 knock-down prevents ERK1/2 activation and abolishes human hepatocarcinoma growth in vitro and in vivo. Int J Cancer 126: 1367-1377, 2010

30. Guo L, Guo Y, Xiao S and Shi X: Protein kinase p-JNK is correlated with the activation of AP-1 and its associated Jun family proteins in hepatocellular carcinoma. Life Sci 77 1869-1878, 2005.
31. Min L, He B, and Hui L: Mitogen-activated protein kinases in hepatocellular carcinoma development. Semin Cancer Biol 21: 10-20, 2011.

32. Simard JC, Girard D and Tessier PA: Induction of neutrophil degranulation by S100A9 via a MAPK-dependent mechanism. J Leukoc Biol 87: 905-914, 2010.

33. Sunahori K, Yamamura M, Yamana J, et al: The S100A8/A9 heterodimer amplifies proinflammatory cytokine production by macrophages via activation of nuclear factor kappa $\mathrm{B}$ and p38 mitogen-activated protein kinase in rheumatoid arthritis. Arthritis Res Ther 8: R69, 2006.

34. Ehlermann P, Eggers K, Bierhaus A, et al: Increased proinflammatory endothelial response to S100A8/A9 after preactivation through advanced glycation end products. Cardiovasc Diabetol 5: $6,2006$.

35. Hiwatashi K, Ueno S, Abeyama K, et al: A novel function of the receptor for advanced glycation end-products (RAGE) in association with tumorigenesis and tumor differentiation of HCC. Ann Surg Oncol 15: 923-933, 2008.

36. Hoefen RJ and Berk BC: The role of MAP kinases in endothelial activation. Vascul Pharmacol 38: 271-273, 2002.

37. Lee HC, Tian B, Sedivy JM, Wands JR and Kim M: Loss of Raf kinase inhibitor protein promotes cell proliferation and migration of human hepatoma cells. Gastroenterology 131: 1208-1217, 2006

38. Montesano R, Soriano JV, Hosseini G, Pepper MS and Schramek H: Constitutively active mitogen-activated protein kinase kinase MEK1 disrupts morphogenesis and induces an invasive phenotype in Madin-Darby canine kidney epithelial cells. Cell Growth Differ 10: 317-332, 1999.

39. Behren A, Binder K, Vucelic G, et al: The p38 SAPK pathway is required for Ha-ras induced in vitro invasion of NIH3T3 cells. Exp Cell Res 303: 321-330, 2005.

40. Huang X, Chen S, Xu L, et al: Genistein inhibits p38 map kinase activation, matrix metalloproteinase type 2 , and cell invasion in human prostate epithelial cells. Cancer Res 65: 3470-3478, 2005.

41. Ringshausen I, Dechow T, Schneller F, et al: Constitutive activation of the MAPkinase p38 is critical for MMP-9 production and survival of B-CLL cells on bone marrow stromal cells. Leukemia 18: 1964-1970, 2004. 